\title{
ARTICLE
}

\section{A feasibility pilot using telehealth videoconference monitoring of home-based NMES resistance training in persons with spinal cord injury}

\author{
Ashraf S Gorgey ${ }^{1,2}$, Robert M Lester ${ }^{1}$, Rodney C Wade, Refka E Khalil ${ }^{1}$, Rehan K Khan ${ }^{3}$, Melodie L Anderson ${ }^{1}$ and Teodoro Castillo ${ }^{1}$
}

INTRODUCTION: The objective of the study was to investigate the feasibility and initial efficacy of telehealth communication in conjunction with surface neuromuscular electrical stimulation (NMES) resistance training (RT) to induce muscle hypertrophy. MATERIALS AND METHODS: This was a home-based setting of within-subject control design of trained vs controlled limbs. Five men with chronic ( $>1$ year postinjury) motor-complete spinal cord injury $(\mathrm{SCl})$ participated in a twice-weekly telehealth videoconference program using home-based NMES-RT for 8 weeks. Stimulation was applied to the knee extensor muscle group of the trained leg, while the untrained leg served as a control. Participants received real-time feedback to ensure a proper setup of electrodes and stimulator to monitor subject safety throughout the entire training session. Magnetic resonance imaging was used to measure cross-sectional areas (CSAs) and intramuscular fat (IMF) of the whole thigh and individual muscle groups. Average two-way travel time, distance traveled in miles and total cost of gas per mile were calculated.

RESULTS: Participants had $100 \%$ compliance. Trained whole and absolute knee extensor muscle CSA increased by $13 \%(P=0.002)$ and $18 \%(P=0.0002)$, with no changes in the controlled limb. Absolute knee flexor and adductor CSAs increased by $3 \%(P=0.02)$ and 13\% $(P=0.0001)$, respectively. Absolute whole thigh and knee extensor IMF CSAs decreased significantly in the trained limb by $14 \%(P=0.01)$ and $36 \%(P=0.0005)$, respectively, with no changes in controlled limb.

DISCUSSION: The pilot work documented that using telehealth communication is a safe, feasible and potentially cost-reducing approach for monitoring home-based NMES-RT in persons with chronic SCI. All trained muscles showed detectable muscle hypertrophy with concomitant decrease in ectopic adipose tissue.

Spinal Cord Series and Cases (2017) 3, 17039; doi:10.1038/scsandc.2017.39; published online 29 June 2017

\section{INTRODUCTION}

Decreasing training frequency may be a successful strategy to guarantee compliance in persons with spinal cord injury (SCl). ${ }^{1}$ Inexpensive home-based telehealth programs have become an available rehabilitation avenue for clinicians to overcome adherence and commitment problems following $\mathrm{SCl}$. These telehealth programs may reduce the heavy economic burden that $\mathrm{SCl}$ imposes on the health-care system. ${ }^{2-4}$ The increasing availability of telehealth programs has allowed clinics and other facilities to better connect with patients and provide services via internet, mobile and video technologies. ${ }^{5-6}$ However, the efficacy of telehealth home-based exercise programs within the $\mathrm{SCl}$ population has been less validated. The use of telehealth communication for the purpose of $\mathrm{SCl}$ rehabilitation may address several of the barriers associated with developing a successful long-term exercise protocol, including the costs of exercise facilities, limited access and lack of adequate transportation.

Electrically evoked resistance training (RT) using surface neuromuscular electrical stimulation (NMES), (Dudley's protocol), has proven to be an effective intervention to evoke muscle hypertrophy in persons with $\mathrm{SCl}^{7-12}$ Several studies indicated that RT, twice weekly from 8 to 16 weeks, increased muscle size significantly compared to pretraining cross-sectional areas (CSAs). ${ }^{7-12}$ Dudley et al. ${ }^{7}$ showed that 8 weeks of twice-weekly
NMES-RT restored knee extensor muscle size to $75 \%$ of what it was 6 weeks post-SCl. Mahoney et $a l^{8}{ }^{8}$ demonstrated that 12 weeks of training increased skeletal muscle size by $>40 \%$ and improved glucose tolerance years after injury. A follow-up study reported that fatigue resistance of the trained knee extensors increased by $33 \%$ after completing 18 weeks of home-based NMES-RT. ${ }^{9}$ Ryan et al. ${ }^{11}$ noted an improvement in mitochondrial capacity by $25 \%$ following 16 weeks twice weekly of NMES-RT. Additionally, Gorgey et al. ${ }^{10}$ showed that 12 weeks of twice-weekly NMES-RT elicited a $>35 \%$ increase in skeletal muscle size, decreased intramuscular fat (IMF) and visceral adipose tissue, increased insulin sensitivity and increased insulin growth factor-1.

The preservation of skeletal muscle integrity is vital for the proper functioning of cellular and whole-body metabolism. Maintaining the integrity of lean mass below the level of injury is associated with improvement in parameters of body composition, metabolic health and, most recently, mitochondrial density and activity. ${ }^{10,13}$ In order to achieve robust increases in muscle size and function, persons with $\mathrm{SCl}$ must adhere to a long-term exercise program. The literature highlights the need for continuous and possibly life-long training strategies following chronic SCl. Gorgey et al. ${ }^{14}$ showed that, following 16 weeks of functional electrical stimulation, leg lean mass increased significantly by $9 \%$ and later decreased by $16 \%, 2.5$ years after cessation of the exercise program. A single subject case report

\footnotetext{
${ }^{1}$ Spinal Cord Injury and Disorders Service, Department of Veterans Affairs, Hunter Holmes McGuire VAMC, Richmond, VA, USA; ${ }^{2}$ Department of Physical Medicine and Rehabilitation, Virginia Commonwealth University, Richmond, VA, USA and ${ }^{3}$ Radiology Service, Hunter Holmes McGuire VA Medical Center, Richmond, VA, USA. Correspondence: AS Gorgey (ashraf.gorgey@va.gov) 
Table 1. Physical characteristics of 5 men with motor-complete $\mathrm{SCl}$ prior participating in 8 weeks home-based telehealth training

\begin{tabular}{|c|c|c|c|c|c|c|c|c|c|c|c|}
\hline & Age (years) & Weight (kg) & Height (m) & $B M I\left(\mathrm{~kg} \mathrm{~m}^{-2}\right)$ & LOI & TSI & AIS & Ethnicity & Trained leg & Weeks of training & Maximum weight (Ibs) \\
\hline 002 & 18 & 75 & 1.84 & 22 & C6 & 2 & B & W & Right & 8 & 14 \\
\hline 004 & 39 & 102 & 1.74 & 33.7 & T11 & 2 & A & AA & Left & 8 & 6 \\
\hline 005 & 49 & 82 & 1.69 & 28.7 & T6 & 7 & B & AA & Right & 8 & 2 \\
\hline Mean \pm s.d. & $36 \pm 12$ & $82.5 \pm 15$ & $1.78 \pm 6$ & $26 \pm 5$ & C6-T11 & $7 \pm 5$ & & & & & $6.5 \pm 5.7$ \\
\hline
\end{tabular}

Abbreviations: AIS, American spinal cord injury Impairment Scale; BMI, body mass index; LOI, level of injury; SCI, spinal cord injury; TSI, time since injury.

suggested that a decrease in training volume to once weekly for 12 weeks may still result in a $7 \%$ increase in leg lean mass from 11 to $11.8 \mathrm{~kg} .{ }^{1,10}$ However, it was clear that the gain in leg lean mass was almost $50 \%$ lower than when the frequency of NMES-RT was set twice weekly. ${ }^{1}$

The purpose of the current study was to demonstrate the efficacy of using a home-based videoconferencing system to monitor training using surface NMES-RT, while providing real-time feedback for the added safety of each participant. Our ultimate objective was to provide a real-time feedback in order to introduce a practical rehabilitation strategy that ensures independency in the setup, maintains compliance and effectively induces skeletal muscle hypertrophy in persons with $\mathrm{SCl}$. The potential cost-effectiveness of using the telehealth program was also demonstrated via measuring travel time, distance and total cost of gas per mile. This data may implicate additional benefits to mitigate recognized barriers associated with long-term exercise compliance after $\mathrm{SCl}$.

\section{METHODS}

Five men with traumatic motor-complete $\mathrm{SCl}$ (C5-T11, American Spinal Injury Association A-B) participated in the current study. The physical characteristics of the five participants are listed in Table 1. The study was conducted at the McGuire Veterans Affairs Medical Center and all participants were asked to read and sign consent forms that were approved by the local ethics committee. The study procedures were conducted according to the Declaration of Helsinki.

The five participants were part of a clinical trial (NCT01652040) investigating the effects of NMES-RT and testosterone replacement therapy on body composition and metabolic profile in persons with $\mathrm{SCl}$ (data not shown). Eligibility criteria of the clinical trial were as follows: male; age 18-50 years; > 1 year postinjury; C5-L2; motor-complete American spinal cord injury Impairment Scale A or $\mathrm{B}$; and body mass index $<30 \mathrm{~kg} \mathrm{~m}^{-2}$. Participants with experience in the application of NMES-RT $(n=11)$ from the parent trial were approached to participate in the current trial. ${ }^{15}$ Five participants were accepted on the basis of first-come-first-serve to participate in an optional 8-week telehealth continuation in a homebased setting. The washout period was designed to eliminate the training effect of participating in the parent clinical trial. Three participants were allowed 1 year following the termination of the initial clinical trial as a washout period before participating in the telehealth NMES training program. Participants 2 and 4 were only allowed a washout period of 2 weeks and 2 months, respectively, owing to school scheduling and other family commitments. The rationale of the washout period is to allow enough time to washout any training effects on muscle mass from participating in the parent clinical trial. ${ }^{14}$

\section{Study design}

The age range of the five participants was $18-50$ years. Eligible participants had a chronic motor-complete SCl (American spinal cord injury Impairment Scale A or B), in order to exclude the inevitable variability of body composition changes that would have been found in individuals with motor-incomplete SCl. Eligible participants were also capable of producing a visible tetanic contraction of the knee extensor muscle group in response to surface NMES.

All participants had no previous history of orthopedic, cardiovascular or metabolic conditions. Participants with preexisting medical conditions (uncontrolled hypertension, uncontrolled hyperglycemia or a hemoglobin A1C level >7.0, chronic arterial diseases, recent deep vein thrombosis, uncontrolled autonomic dysreflexia, severe spasticity, fractures or history of fractures, pressure ulcers greater than grade II, documented osteoporosis, uncompensated hypothyroidism, renal disease) were excluded from the study.

Each training session was continuously monitored by research staff using the Cisco Jabber Video for Telepresence Software. Live video communication allowed researchers to provide real-time feedback to ensure proper placement of electrodes, correct connection of cables and a full range of motion during exercise. While seated in their wheelchair, a unilateral training of one knee extensor (right or left) muscle group was performed using ankle weights, while the untrained knee extensor served as a control. The design of training one limb has been previously considered in persons with $\mathrm{SCl}$ and allows control for genomic, body composition and hormonal variabilities that are likely to impact the outcomes without the necessary large sample size needed to address similar research questions. ${ }^{16,17}$ Only participants with previous experience with surface NMES and ankle weights were considered for the current trial to facilitate the process of electrode placement and donning/doffing of ankle weights.

\section{Familiarization session}

Following consenting, participants underwent medical clearance by an $\mathrm{SCl}$ physician and were invited for a familiarization session conducted by research staff. The session included teaching how to position the electrodes and connect cables to the stimulator, donn/doff ankle weights around the shin, safely turn on a battery operated stimulator (Empi 300PV; Empi Inc., a DJO Global company, St Paul, MN, USA) and ramp up the current until full knee extension was achieved. Following demonstration, each participant was allowed the opportunity to practice for 10-15 min and to ask any questions. Patients/caregivers were then provided an orientation to the Cisco Jabber Video for Telepresence Software (Cisco systems, Inc. San Jose, CA, USA) program features, including troubleshooting and review of Emergency Management Systems protocol, presented by the $\mathrm{SCl}$ telehealth coordinator prior to connection with the provider. The review process along with software program orientation was documented in the participant's electronic health record. ${ }^{5,6}$

Participants were then scheduled for a clinical video teleconference (CVT) RT session after downloading the software on their personal laptops equipped with a standard web camera., ${ }^{5,6}$ A confirmation of the appointment was sent via email to both study participants and research staff. Participants were required to confirm the appointment online at least $24 \mathrm{~h}$ prior to their appointment to receive their log-in password into the Cisco Jabber Video for Telepresence Software.

\section{Telehealth NMES training}

In-home video telehealth services in the VA (known as VA Video Connect) was conducted under the guidance of the office of connected care with management and oversight via the VISN telehealth manager and facility telehealth coordinator. The telehealth program is located within the Spinal Cord Injury/Disorders (SCI/D) system of care., 5 The SCI/D telehealth coordinator conducted a selection-consideration review for each participant and ensured that each participant agreed to use telehealth services for training, reviewed a clinical 'video into home' worksheet and possessed the appropriate technology, including a personal web camera, computer, broadband internet connection and valid email address, to obtain log-in credentials for the in-home Cisco Jabber Video Software. 


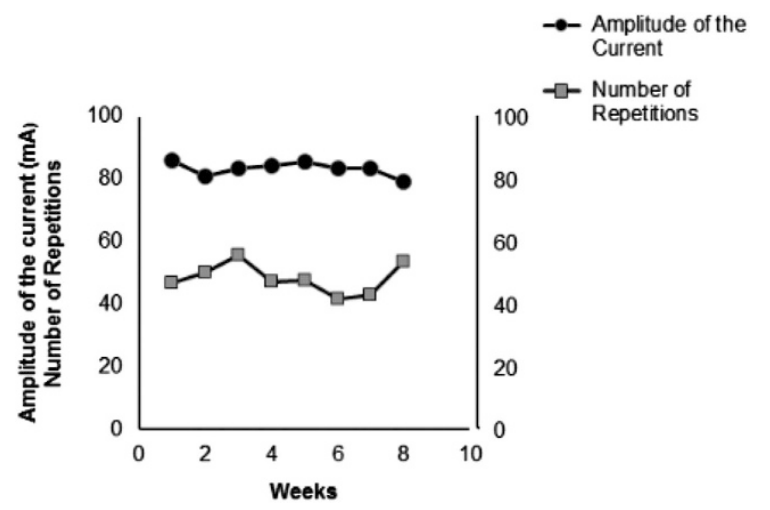

Figure 1. Progression in the amplitude of the current and number of repetitions over the course of 8 weeks in 5 men with motorcomplete $\mathrm{SCl}$.

All participants agreed to provide a private, quiet environment that was conducive to conduct in-home training that was also accessible for Emergency Management Systems in the event of an emergency. At the beginning of each session, research staff obtained the address and telephone number of participant and others in the home during the training session, which would be important in the event of an emergency during the CVT. In the event of an emergency, research staff could contact their local medical/telehealth emergency resources for back-up or the national study participant's crisis line for additional assistance. Certain features were important to be tested in the home prior to starting the exercise training session including the microphone and speakers, as well as the web camera of both study participant and research staff computers. Occasionally, participants reported technological problems on their end of CVT. In the event of communication problems, research staff contacted study participant via telephone call and attempted to resolve the technical issue.

Participants were instructed to be ready 20-30 min prior to the CVT call by placing electrodes, ensuring the batteries of the stimulator were charged overnight, connecting the electrodes to the stimulator via the cables and positioning the ankle weights. After establishing an internet connection, researchers checked to ensure a proper placement of electrodes, connection of stimulator cables and positioning of ankle weights. Once the setup was complete, the participant increased the current to bring the leg into full knee extension and then reported the current in milliamps $(\mathrm{mA})$ to research staff. The participant's goal was to perform 3 sets of 10 repetitions. Each exercise training session was conducted for at least $30 \mathrm{~min}$ with 2-3 min of resting between each set and $5 \mathrm{~s}$ between each repetition. At the end of each session, research staff confirmed the following appointment. Participants were reminded to confirm the appointment online $24 \mathrm{~h}$ in advance in order to access their password log-in credentials.

The training protocol was conducted twice weekly for 8 weeks. ${ }^{7-12}$ During the first week, no ankle weights were worn until the participant achieved 3 sets (30 repetitions) of full knee extensions against gravity. ${ }^{8-10}$ The weights were progressively increased by $2 \mathrm{lbs}$ on a weekly basis. ${ }^{10}$ A portable Empi 300PV stimulator was used along with large conductive adhesive gel electrodes $(8 \times 10 \mathrm{~cm})$. Parameters were preset and locked at squared pulse, frequency of $30 \mathrm{~Hz}$ and $400-\mu$ s pulse duration. ${ }^{7-12}$ The current $(0-100 \mathrm{~mA})$ was gradually increased to activate knee extensors to move the leg and ankle weights dynamically into a concentric (against gravity)/eccentric (with gravity) paradigm. After full knee extension was maintained for $3 \mathrm{~s}$, the current was slowly decreased and the leg was returned to the starting position. ${ }^{7-12}$ Knee extensor fatigue was determined as the number of repetitions out of 30 for each leg $>75 \%$ of knee extension full range of motion (1). Failure of the leg to move $>25 \%$ of range of motion for two subsequent repetitions resulted in termination of the training set. The allocation of the trained limb (right or left) was based on the criteria of which side needs the least amount of the current amplitude to evoke full knee extension.

\section{Estimate of travel costs}

Using the Google map software, we estimated each participant's two-way travel time, distance traveled in miles and total cost of gas per mile
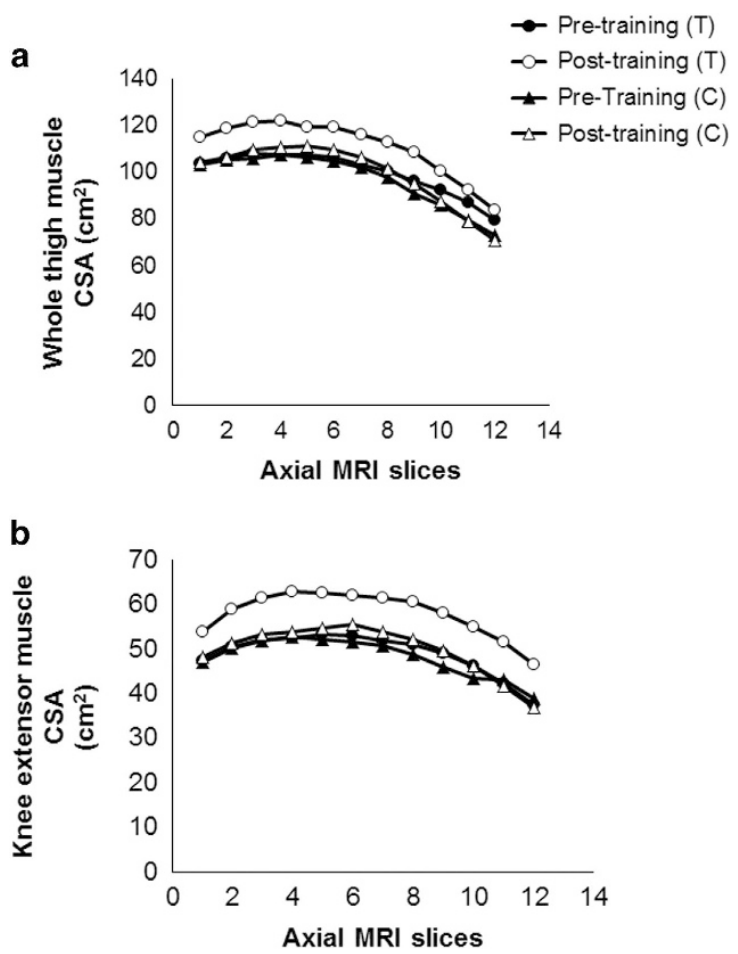

Figure 2. The average of (a) whole thigh and (b) knee extensors skeletal muscle in individuals with $\mathrm{SCl}$. Slice 1 represents the first image distal to the gluteus maximums muscle and slice 12 is the last image towards the knee joint. \#Significant difference between groups $P<0.05$. (T: trained limb and C: control limb).

according to the US federal rate in 2015 ( 23 cents per mile) for medical purpose.

\section{Magnetic resonance imaging}

Whole thigh magnetic resonance imaging (MRI) scans were conducted at baseline and 1 week postintervention to measure skeletal muscle CSAs. ${ }^{8-10}$ Transaxial images, $8 \mathrm{~mm}$ thick and $4 \mathrm{~mm}$ apart, were obtained from the hip to the knee joints using a regional body coil with a 1.5-T magnet (repetition time $550 \mathrm{~ms}$, echo time $14 \mathrm{~ms}$, field of view $20 \mathrm{~cm}$, matrix size $256 \times 256$ ). Images were analyzed by tracing the outer perimeter of the thigh muscle groups using the computer program Win Vessel (Ronald Meyer, Michigan State University, East Lansing, MI, USA). ${ }^{8-10}$ Images were analyzed in a blinded manner by research staff to determine skeletal muscle CSAs for whole thigh (muscle and subcutaneous adipose tissue (SAT)), whole thigh muscle (absolute muscle CSA after excluding IMF), knee extensors, knee flexors and hip adductors before training and 1 week after the last training session. ${ }^{1,10,18}$ Briefly, the outer perimeter of the thigh muscle group was traced and pixel signal intensity within this region was automatically determined. ${ }^{1,10,18}$ Next, a bimodal histogram of two peaks was plotted. The first peak represented muscle and the second represented fat. ${ }^{18}$ A midpoint was calculated by averaging the two peaks. Midpoints of five consecutive MRI images were calculated and subsequently averaged. This average, also called the 'magic number' allows for the separation of muscle and fat pixels based on signal intensity. Once the total number of muscle and IMF pixels were known, further calculations were conducted.

\section{Outcome measures}

Exercise adherence. Exercise adherence was measured as the percentage of sessions completed out of the total recommended sessions ( 16 total sessions) completed over the 8 weeks of NMES-RT.

Muscle and IMF CSAs. The muscle and IMF CSAs were calculated using the following equations: muscle $\operatorname{CSA}\left(\mathrm{cm}^{2}\right)=$ total number of pixels of muscle $\times(\text { field of view/matrix size })^{2}$; IMF CSA $\left(\mathrm{cm}^{2}\right)=$ total number of pixels of IMF $\times$ (field of view/matrix size $)^{2}$. Muscle and IMF CSAs were calculated 
Table 2. Comparisons of the trained vs controlled limbs using mean difference and percentage difference of the thigh, knee extensor, flexor and hip adductor muscle CSAs as well as intramuscular fat (IMF) across MRI slices in persons with SCI

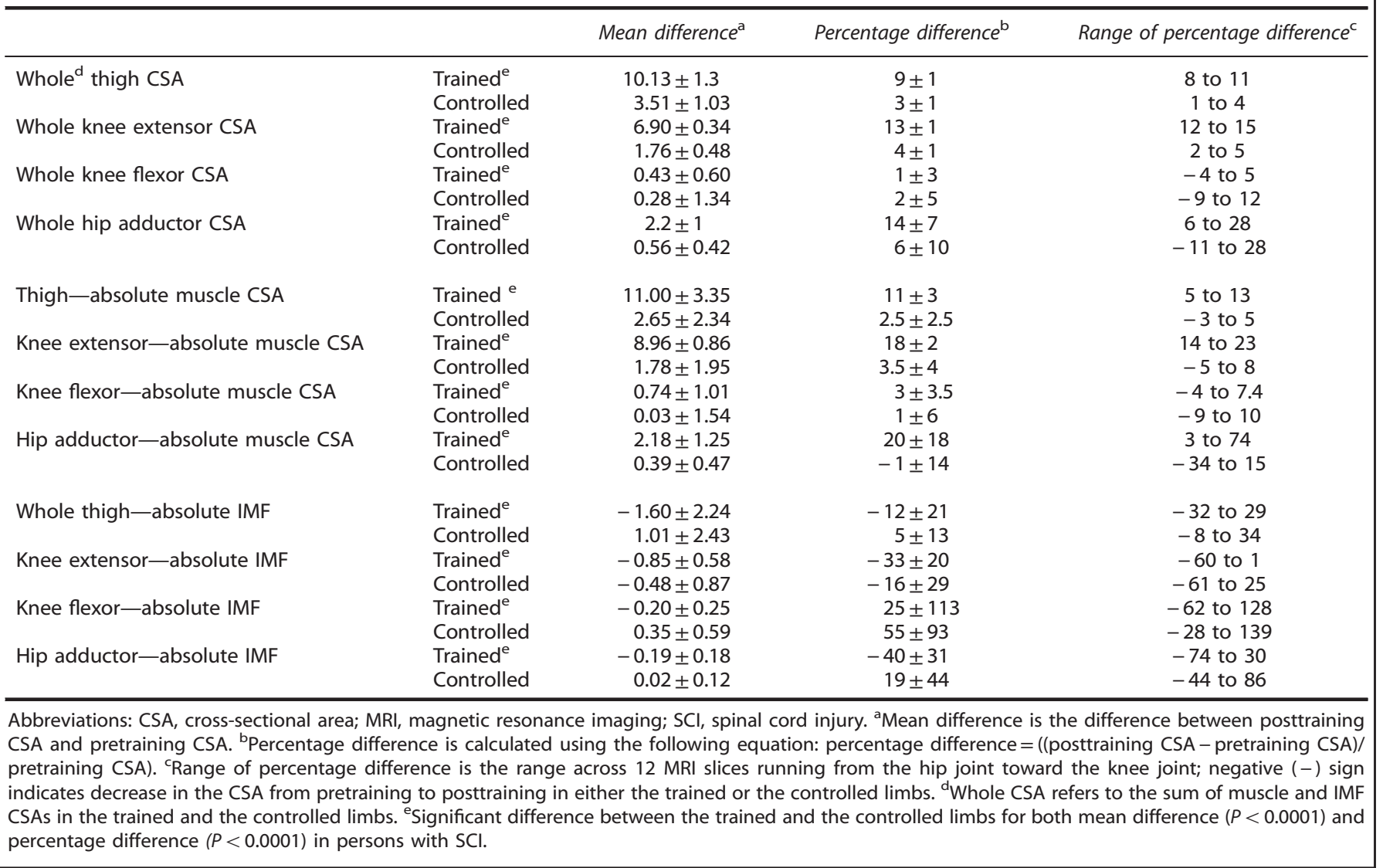

for the whole thigh, whole thigh muscle, knee extensors, knee flexors and hip adductors before training and 1 week after the last training session. Muscle CSA and IMF values were determined by averaging 12-13 MRI slices. SAT CSA was considered as the area between the muscle border and the outer perimeter of the thigh. ${ }^{1,10,18}$

Travel cost. Estimated two-way travel costs were also calculated using the Google map software. Determining the average cost of travel was important to begin to develop an understanding of certain financial barriers to long-term fitness within the $\mathrm{SCl}$ population.

\section{Statistical analysis}

Assumption of normality was tested using Shapiro-Wilk tests as well as $\mathrm{Q}-\mathrm{Q}$ plots. The unilateral stimulation of one knee extensor muscle group (the other serving as a control), allowed for control of the variability that may exist among individuals with $\mathrm{SCl}$ and allowed for direct comparisons between the trained leg and the controlled leg of the same participant. Paired $t$-tests were used to determine the differences in whole thigh skeletal muscle CSAs, individual muscle group CSAs (knee extensors, flexors and hip adductors), IMF and percentage IMF following the 8-week intervention between he trained and the controlled (untrained) legs. Independent $t$-tests were used to test the difference in mean difference and percentage difference between the trained and the controlled limbs. Statistical analyses were performed using IBM-SPSS version 23.0 (SPSS, Chicago, IL, USA). All values are presented in mean \pm s.d. and statistical significance was set at $a<0.05$.

\section{RESULTS}

The physical characteristics of the five participants are presented in Table 1. All muscle data were normally distributed as determined by non-significant Shapiro-Wilk tests $(P>0.05)$ and the Q-Q plots. Four participants completed 16 visits in
8 weeks with a compliance rate of $100 \%$. Participant 3 completed 6 weeks of training and he then developed bilateral iliac clots. The participant agreed to have his MRI captured to evaluate the effects of training on muscle size after obtaining medical clearance.

On average, participants were able to lift $6.5 \pm 5.7 \mathrm{lbs}$ (range $0-14 \mathrm{lbs}$ ) of ankle weights by the last week of training. The amplitudes of the current $(0-100 \mathrm{~mA})$ required to evoke full knee extension as well as the number of repetitions ( 3 sets $\times 10$ repetitions $\times 2$ visits weekly $=60$ reps) achieved over the course of 8 weeks are presented in Figure 1. On week 8, participants were able to complete $89 \%$ (54/60 reps) of the assigned repetitions.

\section{Skeletal muscle CSA}

Whole thigh muscle (muscle+IMF) CSA increased significantly by $9 \%$ from $111 \pm 10$ to $121 \pm 11 \mathrm{~cm}^{2}(P=0.03)$ in the trained leg, with no changes in the controlled limb $\left(108 \pm 11-112 \pm 11 \mathrm{~cm}^{2}\right.$; $3.2 \% ; P=0.43$ ) following 8 weeks of training. Whole thigh absolute muscle CSA increased by $11 \%$ from $100 \pm 9$ to $111 \pm 11 \mathrm{~cm}^{2}(P=0.02)$, with no changes in the controlled limb $\left(97 \pm 11-99 \pm 13 \mathrm{~cm}^{2} ; P=0.6\right)$ (Figure 2a).

The trained whole knee extensor muscle CSA increased by $13 \%$ from $52.5 \pm 5$ to $59.4 \pm 5 \mathrm{~cm}^{2} \quad(P=0.002$; Figure $2 \mathrm{~b})$, with no changes in the controlled limb $\left(49.4 \pm 5-51 \pm 5 \mathrm{~cm}^{2} ; P=0.3\right)$. Absolute knee extensor muscle CSA after excluding IMF increased by $18 \%\left(49 \pm 5-58 \pm 5 \mathrm{~cm}^{2} ; P=0.0002\right)$, with no changes in the controlled limb. Whole knee flexor muscle CSA increased by $2 \%\left(21.7 \pm 8-22.2 \pm 8 \mathrm{~cm}^{2} ; P=0.03\right)$, with no changes in the controlled limb. Absolute knee flexor CSA after excluding IMF increased by $3.5 \%$ from $21 \pm 8$ to $22 \pm 9 \mathrm{~cm}^{2} \quad(P=0.003)$, with 
a

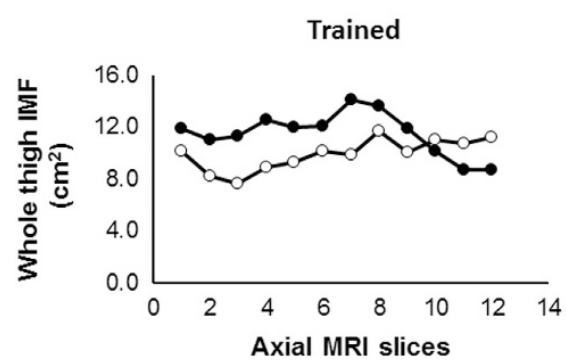

b

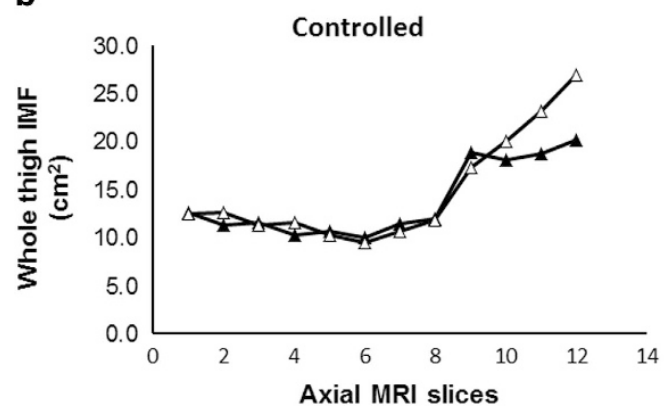

c

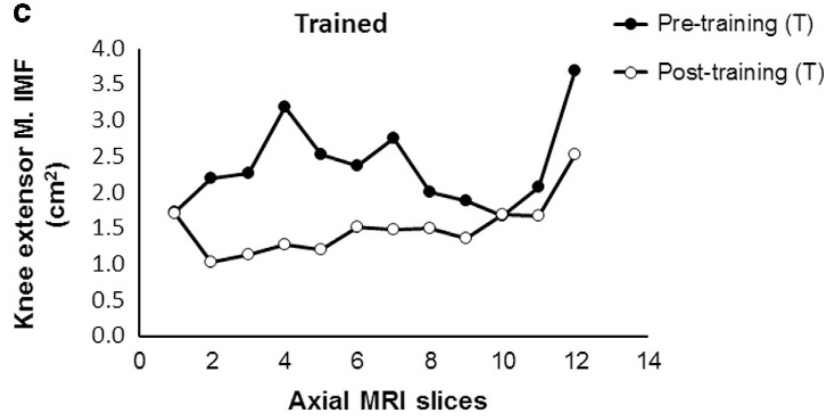

d

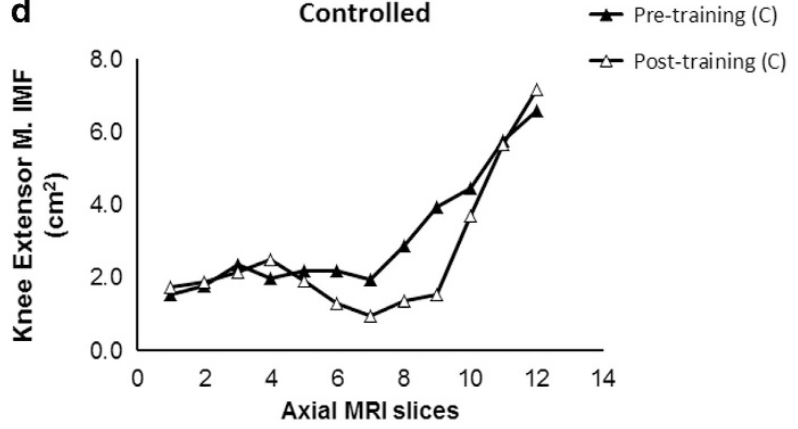

Figure 3. The average of whole thigh (a) trained vs (b) controlled and knee extensor IMF (c) trained vs (d) controlled in individuals with SCI. Slice 1 represents the first image distal to the gluteus maximums muscle and slice 12 is the last image towards the knee joint. \#Significant difference between groups $P<0.05$. (T: trained limb and C: control limb).

no changes in the controlled limb. Whole hip adductor CSA increased by $12 \%\left(16.7 \pm 12.5-18.7 \pm 13.5 \mathrm{~cm}^{2} ; P=0.0003\right)$, with no changes in the controlled limb. Absolute hip adductor CSA after excluding IMF increased by $13 \%$ from $16.3 \pm 12.3$ to $18.4 \pm 13.5 \mathrm{~cm}^{2}$ $(P=0.0001)$. Table 2 presents the comparisons between the trained vs controlled limbs of the whole and absolute muscle CSAs using either mean difference or percentage difference across MRI slices.

Intramuscular fat

Absolute whole thigh IMF decreased significantly in the trained limb by $14 \%\left(11.5 \pm 1.6-10 \pm 1.2 \mathrm{~cm}^{2} ; P=0.01\right.$; Figure $\left.3 a\right)$, with no changes in the controlled limb $\left(14 \pm 4-15 \pm 5.7 \mathrm{~cm}^{2} ; P=0.6\right.$; Figure $3 \mathrm{~b}$ ). Whole thigh percentage IMF decreased from $10.4 \pm 1.1$ to $8.5 \pm 2.3 \%$ following 8 weeks of training $(P=0.02)$. Absolute whole knee extensor IMF decreased significantly in the trained limb by $36 \%\left(2.4 \pm 0.6-1.5 \pm 0.4 \mathrm{~cm}^{2} ; P=0.0005\right.$; Figure $\left.3 \mathrm{c}\right)$, with no changes in the controlled limb $\left(3.12 \pm 1.7\right.$ to $2.6 \pm 1.9 \mathrm{~cm}^{2} ; P=0.5$; Figure $3 \mathrm{~d}$ ). Knee extensor percentage IMF decreased from $4.5 \pm 1.5$ to $2.6 \pm 0.95 \%$ following 8 weeks of training $(P=0.0008)$, with no changes in the controlled limb $(6.7 \pm 4.4-5.5 \pm 4.8 \%$.; $P=0.55)$

Absolute whole knee flexor IMF decreased significantly in the trained limb by $20 \%\left(1 \pm 0.7-0.77 \pm 0.5 \mathrm{~cm}^{2} ; P=0.02\right)$, with no changes in the controlled limb $(P=0.15)$. Absolute adductor IMF decreased significantly in the trained limb by $38.5 \%$ $\left(0.5 \pm 0.17-0.31 \pm 0.16 \mathrm{~cm}^{2} ; P=0.004\right)$, with no change in the controlled limb $\left(0.32 \pm 0.12-0.35 \pm 0.11 \mathrm{~cm}^{2} ; P=0.54\right)$. There were no changes in percentage IMF of knee flexors or hip adductors following training. Mean difference and percentage difference of IMF between trained and controlled limbs are presented in Table 2.

\section{Estimated travel costs}

The average two-way travel time was $65 \pm 30 \mathrm{~min}$ (36-110 min). The estimated distance traveled in miles was $33 \pm 27$ miles (10-77 miles) and total cost of gas per visit was $\sim \$ 7.50$ per visit. Therefore, the total travel cost was estimated at $\$ 120 \pm 100$ for 8 weeks of training. The duration of the CVT session was 30-40 min per participant and, owing to the home-setting, required no additional travel expenses.

\section{DISCUSSION}

The current study was undertaken to determine the efficacy and safety of using video teleconferencing to monitor 8 weeks of NMES-RT in persons with SCl. The goal of our program was to ensure independency, compliance with the established protocol and effectively evoke skeletal muscle hypertrophy, while providing a real-time feedback. The findings revealed that using live videoconferencing was an effective and safe strategy to conduct an 8-week NMES-RT program designed to evoke muscle hypertrophy and decrease IMF after SCl.

Atrophy of paralyzed muscle has been associated with dysfunction of the metabolic profile, increasing the risks of glucose intolerance, insulin resistance and type II diabetes after SCI. ${ }^{1,10,19,20}$ Additionally, muscle atrophy has been shown to be associated with increased IMF, further heightening the risks of developing glucose intolerance and insulin resistance. ${ }^{19,20}$ The accumulation of ectopic adipose tissue may interfere with insulin signaling and contribute to increased circulating triglycerides and free fatty acids, which increase the risk of cardiovascular disease. ${ }^{19,20}$ Moreover, skeletal muscle atrophy following chronic $\mathrm{SCl}$ has been associated with reduced mechanical loading of bone and subsequent decline in bone mineral density. ${ }^{16,17}$ Therefore, the preservation of skeletal muscle integrity has significant clinical implications for this population.

The use of real-time videoconferencing has improved the quality of NMES training in persons with $\mathrm{SCl}$ by providing each participant with a live feedback on several aspects, including quality of muscle contraction, functional movement of the lower extremity and muscle fatigue. The quality of our videoconferencing approach was evaluated in a number of ways, including the ability of participants to independently and correctly place electrodes, position ankle weights, connect NMES cables and safely utilize the NMES unit to gradually increase the current until 
achieving full leg extension. The precision of participants to accomplish these tasks improved throughout the intervention and a maximum adherence of $100 \%$ was achieved by four of the five participants, despite day-to-day activities. Verbal communication indicated that training was convenient and safely executed throughout the 8-week intervention. There is limited evidence of comparable protocols using videoconferencing to monitor home-based NMES-RT after SCI. A similar home-based NMES-RT study conducted twice weekly for 18 weeks demonstrated a $60 \%$ reduction in muscle fatigue and $100 \%$ compliance; however, participants were monitored via telephone. ${ }^{9}$ The study did not provide any measure to ensure that electrodes were correctly positioned or that the stimulation parameters were appropriately adjusted during NMES training.

\section{NMES training and muscle hypertrophy}

A battery-operated portable stimulator was used to evoke skeletal muscle hypertrophy. A previous study noted no difference in peak torque of the knee extensor muscle group between a standard clinical NMES stimulator and the portable stimulator. ${ }^{21}$ An increase in muscle size was noticeable not only in the activated knee extensors but also in the hip adductor and knee flexor groups. Similar to previous studies, skeletal muscle hypertrophy in the whole thigh, knee extensor, knee flexor and adductor groups were noted; however, the extent of hypertrophy was limited compared with what has been previously reported. ${ }^{7,10}$ The discrepancy in the extent of muscle hypertrophy observed can be attributed to the difference in the upper limit of the current amplitude between the Empi unit $(100 \mathrm{~mA})$ and previously used units $(200 \mathrm{~mA}){ }^{10}$ Moreover, the duration of the current study ( 8 weeks) was shorter compared with other previously NMES-RT studies (12-16 weeks) after $\mathrm{SCl}$. Higher current amplitude (that is, >100 mA) may be necessary to evoke full leg extension owing to extensive muscle atrophy, infiltration of IMF and the insulator property of SAT that impedes the progression of the current amplitude. ${ }^{2,23}$ Over the course of the training, two participants were able to lift 10 and $14 \mathrm{lbs}$; whereas the other three participants were restricted to only 0-6 lbs, mainly because the current was limited to $100 \mathrm{~mA}$.

\section{NMES training and IMF}

Both absolute and percentage IMF decreased in response to muscle hypertrophy, which suggest improvement in the metabolic flexibility of the trained muscles compared with the controlled limb. Increased IMF has been shown to explain $70 \%$ of the variance in glucose tolerance in persons with chronic SCl. ${ }^{24}$ Decreased IMF has been associated with improvement in insulin sensitivity following 12 weeks of electrically evoked NMES-RT. ${ }^{10}$ Recently, an increase in leg lean mass has been associated with increased mitochondrial mass and a concomitant decrease in IMF. ${ }^{13}$ An increased mitochondrial volume, that utilizes IMF as a major source of energy may explain why muscle hypertrophy is accompanied with decreases in IMF. ${ }^{13}$ Contrary to previous and the current findings, ${ }^{10}$ others have shown that 16 weeks of NMES-RT did not influence IMF CSA in persons with motorcomplete $\mathrm{SCl} .{ }^{11}$ The current findings suggest that muscle hypertrophy is likely responsible for the decrease in both absolute and relative IMF.

\section{Contralateral training effects}

Unilateral training showed only muscle hypertrophy in the trained limb without contralateral effects on the untrained limb. Available evidence clearly indicates that ipsilateral strength training results in contralateral strength gains on the non-trained side. ${ }^{25}$ The pooled estimates of 16 studies showed increases in the contralateral strength of $7.6 \%$ or about half the increase in strength of the trained side. ${ }^{26}$ However, these studies have indicated no evidence that unilateral strength training leads to contralateral muscle hypertrophy. ${ }^{26}$ The current study confirmed these findings and showed that unilateral training using surface NMES-RT did not evoke contralateral hypertrophy of the control limb.

\section{Videoconferencing to maximize adherence}

Maintaining a long-term exercise program is necessary to sustain muscle health after $\mathrm{SCl}^{27,28}$ Hicks et al. ${ }^{28}$ noted an $82.5 \%$ exercise adherence rate during a twice-weekly progressive resistive exercise over a 9-month period in persons with $\mathrm{SCl}$. However, the dropout rate was $47 \%$ over the 9-month program, ${ }^{28}$ suggesting that better strategies need to be implemented to ensure long-term retention in exercise programs. In a similar home-based study, Dolbow et al. ${ }^{27}$ incentivized continued adherence of weekly functional electrical stimulation cycling by notifying that, if individual participation was close to the desired three sessions per week for the first 8 weeks, the cycle would be purchased for their continued long-term home use. Notably, the adherence dropped from $72 \%$ to $63 \%$ following 8 weeks of training, most likely due to the lack of incentive after the 8-week decision to purchase cycle. ${ }^{27}$ We noted an $100 \%$ adherence rate over the course of the 8-week training. Therefore, a home-based exercise training program under live supervision may provide an alternative approach for long-term adherence and retention for persons with $\mathrm{SCl}$.

\section{Exercise barriers after $\mathrm{SCl}$}

Previously, exercise barriers have been highlighted in persons with $\mathrm{SCl}^{29}$ These barriers may include lack of access to SCl-equipped facilities, lack of accessible public transportation, limited knowledge on managing persons with $\mathrm{SCl}$ and the associated medical complications, failure to design an appropriate exercise program based on the level of injury and lack of qualified caregivers. ${ }^{29}$ Additionally, over the past two decades, the numbers of days allotted to rehabilitation have declined markedly. ${ }^{30-32}$ A study by Whiteneck et al. ${ }^{31}$ analyzing discharge data of patients with traumatic SCl reported that, from 2005 to 2008, the average number of days spent in a rehabilitation facility decreased by $>50 \%{ }^{30}$ The National Spinal Cord Injury Statistical Center has determined that rehabilitation lengths of stay have decreased from 98 days in the 1970s to 35 days in 2016. ${ }^{31}$ The emergence of telehealth programs may allow for continued rehabilitation after discharge by overcoming the aforementioned exercise and socioeconomic barriers and providing a long-term intervention after $\mathrm{SCl}$.

The costs is notably the greatest barrier limiting access to exercise activities, and as such reducing costs of treatments is a major necessity for members of rural and urban communities. ${ }^{33}$ Our study introduced a method that can attenuate much of the added costs of long-term exercise traditionally provided at community gyms and private facilities. Home-based training described in this study eliminated the need for routine transportation to and from a rehabilitation facility or gym. We estimated the total travel cost to be $\sim \$ 120 \pm 100$ for 8 weeks of training. The duration of the videoconference training session was 30-40 min per participant and one participant performed the training protocol in his home as well as his lunch break during work, suggesting the flexibility of carrying on the training protocol without restriction to a specific location. Additionally, we need to acknowledge that if similar protocols are to be implemented successfully and sustainably the added cost of equipment (for example, personal computer, portable stimulator, gel electrodes) and qualified staff cannot be neglected. Home-based videoconferencing is one aspect of accessible health care that has the potential to reduce costs for members of the $\mathrm{SCl}$ community. 
Limitations

There are few aspects that need to be considered as limitations of the current pilot work. The limited washout period for two of the five subjects was a notable limitation of the current study. The maximum weight (14 lbs) was lifted in week 8 by a participant who engaged in telehealth training after immediately finishing participation in a clinical trial. It is unclear whether the parent clinical trial confounded the results for this participant following his 2-week washout period. Similar to other reports, ${ }^{8-10}$ the training was limited to five participants with motor-complete $\mathrm{SCl}$. The small sample size was chosen primarily because of the robustness of MRI to detect minimal changes in muscle size and IMF. The calculated effect size of whole thigh muscle and knee extensor CSAs based on previous reports was close to 2.7. In terms of the study duration, we have limited our study to 8 weeks; ${ }^{7}$ however, future trials should consider a longer duration to maximize the training effects.

One participant experienced swelling in both legs as result of bilateral iliac clotting in week 6 . We immediately cancelled the training session and asked him to report to his physician to perform a medical exam; his participation in the trial was then terminated. However, it was unclear whether this adverse event was directly related to his participation or if whether stemmed from other unrelated factors. The applicant previously participated in several electrical stimulation training protocols in our laboratory and never experienced any side effects. ${ }^{1,34}$

Future studies should build upon the model presented here by increasing the sample size, extending the duration of training and devising precise methods to determine patient's perceived safety and quality of intervention. Studies should investigate the outcomes of various telehealth strategies to determine the most practical and cost-reducing methods. In terms of practical implementation, this study serves as a model, just beginning to attenuate the barriers to long-term exercise compliance within the $\mathrm{SCl}$ community. An understanding of the factors affecting costs and access is essential to the health and well-being of persons with chronic $\mathrm{SCl}$. As telehealth services evolve and spread to more regions of the globe in the coming years, we can hope that traditional limitations such as travel costs, fear and anxiety associated with traveling and leaving the home and limited access will no longer prevent persons from receiving the life-long health care and exercise needed after $\mathrm{SCl}$.

\section{CONCLUSION}

We have demonstrated that the use of live videoconferencing is an effective and safe strategy to monitor home-based RT using NMES designed to evoke muscle hypertrophy in persons with chronic $\mathrm{SCl}$. The use of a real-time video-monitoring program ensured independence, safety and compliance over the 8-week training. Live feedback ensured that the setup and all aspects of training were carried out precisely as outlined in the protocol to maximize muscle hypertrophy. The unilateral training evoked detectable increases in muscle size without any notable changes in the contralateral controlled limb. This was accompanied with a decrease in both absolute and percentage IMF of the trained limbs. We have demonstrated the potential cost-reducing capacity of such protocols by documenting a reduction in travel time, travel distance and total cost of gas. However, further investigation is needed to determine the efficacy of similar telehealth programs to significantly reduce costs and maintain long-term adherence.

\section{ACKNOWLEDGEMENTS}

We thank the participants who devoted the time and effort to participate in the current case study. We also thank Hunter Holmes McGuire Research Institute and Spinal Cord Injury Services and Disorders for providing the environment to conduct clinical human research trials, as well as, all the MRI technicians who assisted in the process of performing scans. ASG is currently supported by the Department of
Veteran Affairs, Veteran Health Administration, Rehabilitation Research and Development Service (B7867-W) and DoD-CDRMP (W81XWH-14-SCIRP-CTA).

\section{COMPETING INTERESTS}

The authors declare no conflict of interest.

\section{REFERENCES}

1 Gorgey AS, Caudill C, Khalil RE. Effects of once weekly NMES training on knee extensors fatigue and body composition in a person with spinal cord injury. J Spinal Cord Med 2016; 39: 99-102.

2 Dryden DM, Saunders LD, Rowe BH, May LA, Yiannakoulias N, Svenson LW et al. Utilization of health services following spinal cord injury: a 6-year follow-up study. Spinal Cord 2004; 42: 513-525.

3 Houlihan BV, Jette A, Friedman RH, Paasche-Orlow M, Ni P, Wierbicky J et al. A pilot study of a telehealth intervention for persons with spinal cord dysfunction. Spinal Cord 2013; 51: 715-720.

4 Dallolio L, Menarini M, China S, Ventura M, Stainthorpe A, Soopramanien A et al. Functional and clinical outcomes of telemedicine in patients with spinal cord injury. Arch Phys Med Rehabil 2008; 89: 2332-2341.

5 VA Video Connect Operations Manual Supplement (April 2016). Office of Connected Care, Department of Veterans Health Affairs. www.telehealth.va.gov.

6 Emergency Procedures for VA Video Connect Guidance (19 January 2013). Office of Connected Care, Department of Veterans Health Affairs. www.telehealth.va.gov.

7 Dudley GA, Castro MJ, Rogers S, Apple DF Jr. A simple means of increasing muscle size after spinal cord injury: a pilot study. A simple means of increasing muscle size after spinal cord injury: a pilot study. Eur J Appl Physiol Occup Physiol 1999; 80: 394-396.

8 Mahoney ET, Bickel CS, Elder C, Black C, Slade JM, Apple D Jr et al. Changes in skeletal muscle size and glucose tolerance with electrically stimulated resistance training in subjects with chronic spinal cord injury. Arch Phys Med Rehabil 2005: 86: 1502-1504.

9 Sabatier MJ, Stoner L, Mahoney ET, Black C, Elder C, Dudley GA et al. Electrically stimulated resistance training in $\mathrm{SCl}$ individuals increases muscle fatigue resistance but not femoral artery size or blood flow. Spinal Cord 2006; 44: 227-233.

10 Gorgey AS, Mather KJ, Cupp HR, Gater DR. Effects of resistance training on adiposity and metabolism after spinal cord injury. Med Sci Sports Exerc 2012; 44: 165-174.

11 Ryan TE, Brizendine JT, Backus D, McCully KK. Electrically induced resistance training in individuals with motor complete spinal cord injury. Arch Phys Med Rehabil 2013; 94: 2166-2173.

12 Bickel CS, Yarar-Fisher C, Mahoney ET, McCully KK. Neuromuscular electrical stimulation-induced resistance training after SCl: a review of the Dudley Protocol. Top Spinal Cord Inj Rehabil 2015; 21: 294-302.

13 O'Brien LC, Wade R, Segal L, Chen Q, Savas J, Lesnefsky EJ et al. Mitochondrial mass and activity as a function of body composition in individuals with spinal cord injury. Physiol Rep 5: e13080.

14 Gorgey AS, Martin H, Metz A, Khalil RE, Dolbow DR, Gater DR. Longitudinal changes in body composition and metabolic profile between exercise clinical trials in men with chronic spinal cord injury. J Spinal Cord Med 2016; 39: 699-712.

15 Gorgey AS, Khalil RE, Gill R, O'Brien LC, Lavis T, Castillo T et al. Effects of testosterone and evoked resistance exercise after spinal cord injury (TEREX-SCI): study protocol for a randomised controlled trial. BMJ Open 2017; 7: e014125.

16 Shields RK, Dudley-Javoroski S. Musculoskeletal plasticity after acute spinal cord injury: effects of long-term neuromuscular electrical stimulation training. J Neurophysiol 2006; 95: 2380-2390.

17 Shields RK, Dudley-Javoroski S. Musculoskeletal adaptations in chronic spinal cord injury: effects of long-term soleus electrical stimulation training. Neurorehabil Neural Repair 2007; 21: 169-179.

18 Gorgey AS, Shepherd C. Skeletal muscle hypertrophy and decreased intramuscular fat after unilateral resistance training in spinal cord injury: case report. J Spinal Cord Med 2010; 33: 90-95.

19 Gorgey AS, Dolbow DR, Dolbow JD, Khalil RK, Castillo C, Gater DR. Effects of spinal cord injury on body composition and metabolic profile - part I. J Spinal Cord Med 2014; 37: 693-702.

20 Bauman WA, Spungen AM. Carbohydrate and lipid metabolism in chronic spinal cord injury. J Spinal Cord Med 2001; 24: 266-277.

21 Lyons CL, Robb JB, Irrgang JJ, Fitzgerald GK. Differences in quadriceps femoris muscle torque when using a clinical electrical stimulator versus a portable electrical stimulator. Phys Ther 2005; 85: 44-51. 
22 Gorgey AS, Cho GM, Dolbow DR, Gater DR. Differences in current amplitude evoking leg extension in individuals with spinal cord injury. NeuroRehabilitation 2013; 33: 161-170.

23 Gorgey AS, Mahoney E, Kendall T, Dudley GA. Effects of neuromuscular electrical stimulation parameters on specific tension. Eur J Appl Physiol 2006; 97: 737-744.

24 Elder CP, Apple DF, Bickel CS, Meyer RA, Dudley GA. Intramuscular fat and glucose tolerance after spinal cord injury--a cross-sectional study. Spinal Cord 2004; 42: 711-716.

25 Carroll TJ, Herbert RD, Munn J, Lee M, Gandevia SC. Contralateral effects of unilateral strength training: evidence and possible mechanisms. I Appl Physiol 1985; 2006101: 1514-1522.

26 Munn J, Herbert RD, Gandevia SC. Contralateral effects of unilateral resistance training: a meta-analysis. J Appl Physiol 2004; 96: 1861-1866.

27 Dolbow DR, Gorgey AS, Ketchum JM, Moore JR, Hackett LA, Gater DR. Exercise adherence during home-based functional electrical stimulation cycling by individuals with spinal cord injury. Am J Phys Med Rehabil 2012; 91: 922-930.

28 Hicks AL, Martin KA, Ditor DS, Latimer AE, Craven C, Bugaresti J et al. Long-term exercise training in persons with spinal cord injury: effects on strength, arm ergometry, performance and psychological well-being. Spinal Cord 2003; 41: 34-43.

29 Gorgey AS. Exercise awareness and barriers after spinal cord injury. World J Orthop 2014; 5: 158-162.

30 Ottenbacher K, Smith PM, Illig SB, Linn RT, Ostir GV, Granger CV. Trends in length of stay, living setting, functional outcome, and mortality following medical rehabilitation. JAMA 2004; 292: 1687-1695.

31 Whiteneck G, Gassaway J, Dijkers MP, Lammertse DP, Hammond F, Heinemann AW et al. Inpatient and post discharge rehabilitation services provided in the first year after spinal cord injury: findings from the SCIRehab study. Arch Phys Med Rehabil 2011; 92: 361-368.

32 Eastwood E, Hagglund K, Ragnarsson K, Gordon W, Marino R. Medical rehabilitation length of stay and outcomes for persons with traumatic spinal cord injury-1990-1997. Arch Phys Med Rehabil 1999; 80: 1457-1463.

33 Tate D, Forcheimer M. Enhancing community reintegration after inpatient rehabilitation for persons with spinal cord injury. Top Spinal Cord Inj Rehabil 1998; 4: 42-55.

34 Gorgey AS, Harnish CR, Daniels JA, Dolbow DR, Keeley A, Moore J et al. A report of anticipated benefits of functional electrical stimulation after spinal cord injury. J Spinal Cord Med 2012; 35: 107-112. 